www.nature.com/pj

\title{
Effect of the cast-solvent on the morphology of cast films formed with a mixture of stereoisomeric poly(lactic acids)
}

\author{
Yuichiro Koide, Hiroki Ikake, Yoshio Muroga and Shigeru Shimizu
}

Semicrystalline films were prepared by casting chloroform (CHL), acetonitrile (ACN) or tetrahydrofuran (THF) solutions of a mixture (mix-poly(lactic acid) (PLA)) of poly(L-lactic acid) (PLLA) and poly(D-lactic acid) (PDLA). All of the films involve stereocomplex crystals (sc-PLA) of PLLA and PDLA, but no crystals of PLLA or PDLA. The origin of the marked cast-solvent dependence of the morphology was studied by assuming that the structure of Sc-PLA would be constructed by the balance of the interaction energy $E_{1}$ between the PLLA and PDLA chains in solution, and the interaction energy $E_{2}$ between PLLA or PDLA chains and solvent molecules. With the relation between $E_{1}$ and $E_{2}$, the scheme for the formation of sc-PLA was classified into two categories, Category-I and -II. In Category-I, the relation $E_{1}>E_{2}$ holds over the entire concentration range, and in Category-II, the relation $\mathrm{E}_{1}<\mathrm{E}_{2}$ holds over the range from dilute to moderate concentrations. As a result, the data indicate that Category-I would be proper when SC-PLA is formed by casting a THF or ACN solution of mix-PLA, and that Category-II would be proper when Sc-PLA is formed by casting the CHL solution of mix-PLA.

Polymer Journal (2013) 45, 645-650; doi:10.1038/pj.2012.192; published online 28 November 2012

Keywords: cast-solvent; differential scanning calorimetry; poly(lactic acid); small-angle X-ray scattering; stereocomplex; wide-angle X-ray diffraction

\section{INTRODUCTION}

One of the biomass polymers, poly(lactic acid) (PLA), has attracted much attention as an alternative for petroleum-based polymers. ${ }^{1}$ It is well known that PLA has two stereoisomers, poly(D-lactic acid) (PDLA) and poly(L-lactic acid) (PLLA), and their mixture (mixPLA) can be used to construct stereocomplex crystals (sc-PLA). It was noted by Tsuji and $\mathrm{Ikada}^{2}$ that sc-PLA has better thermal and mechanical properties than crystals of PLLA or PDLA, and thus, some semicrystalline polymer films formed with mix-PLA are expected to be usefully employed in various fields.

In general, semicrystalline polymer films are prepared either by melting a polymer at a high temperature and then molding it at a low temperature (melt-method) or by dissolving the polymer in suitable solvents and casting the solution (cast-method). However, the latter method would provide a more varied morphology of the cast films when the cast-solvent(s), original concentration of polymers, rate of vaporization of solvents and/or other variables are suitably chosen.

It is well established that the conformation of PLLA and PDLA in the architecture of sc-PLA is a helical chain, ${ }^{3}$ and their conformation in solution is not a helical chain, but rather, a randomly coiled chain. In chloroform $(\mathrm{CHL})$, they behave as fairly expanded randomly coiled chains, as is implied by the relationship between the intrinsic viscosity and the molecular weight, Equation (1). Accordingly, in the preparation of mix-PLA films with the cast-method, as the solution concentration is raised and/or a mixed solution of PLLA and PDLA is left standing for a longer period of time, the PLLA and PDLA chains start to aggregate, and their chain conformation will gradually be changed to a helical conformation such that helical PLLA and PDLA might construct sc-PLA crystals. The structure of sc-PLA thus formed would be more or less modified from the intact structure of sc-PLA because excessive PLLA and PDLA tend to interfere with constructing the structure, and short helical sequences of PLLA and PDLA tend to pair with each other. Accordingly, it is supposed that the morphology of the film would depend on the type of cast-solvents employed. In past years, many studies ${ }^{2,4-7}$ have been carried out on the morphology of sc-PLA films. However, as far as we know, very few studies have been published on the origin of the cast-solvent dependence of the morphology of a cast film. The present work is devoted to clarifying this topic.

In the present work, mix-PLA films were prepared with CHL, acetonitrile (ACN) or tetrahydrofuran (THF) as the solvent, and the morphology was analyzed by small-angle X-ray scattering (SAXS), wide-angle X-ray diffraction (WAXD) and differential scanning 
calorimetry (DSC). These three cast-solvents were chosen because the films cast with the solvents have quite different appearances and toughness values, as is stated below in the Results and Discussion, suggesting their morphology is quite different from each other. The original chain conformation of PLLA and PDLA in solution and the rate of aggregation of a mixture of PLLA and PDLA were also studied by SAXS and cloud-point measurements, respectively.

\section{EXPERIMENTAL PROCEDURE}

\section{Synthesis and characterization of PDLA and PLLA}

L-lactide (or D-lactide) (20 g) was dissolved in diphenyl ether (0.2 l) and then tin(II) chloride (catalyst) $(0.145 \mathrm{~g})$ was added to the solution. ${ }^{8}$ The ringopening polymerization of each lactide was carried out at $130{ }^{\circ} \mathrm{C}$ for $20 \mathrm{~h}$ under a reduced pressure of $1.5 \mathrm{~mm} \mathrm{Hg}$ After the reaction was completed, the reaction mixture was diluted with CHL (0.2l), and the diluted solution was filtered to remove the catalyst. Then, the filtered solution was poured into methanol (2l) to precipitate the dissolved polymer (PLLA or PDLA). The precipitated polymer was further purified by the solution/precipitation method with CHL as the solvent and methanol as the nonsolvent. The purity of the polymer thus obtained with a yield of $\sim 70 \%$ was proved to be satisfactory for the present purpose from its ${ }^{1} \mathrm{H}-\mathrm{NMR}$ and ${ }^{13} \mathrm{C}-\mathrm{NMR}$ spectra. ${ }^{9}$

The viscosity-averaged molecular weight, $M_{v}$, of PDLA and PLLA was evaluated to be $3.36 \times 10^{4}$ and $3.38 \times 10^{4}$, respectively, by Equation (1). ${ }^{10}$

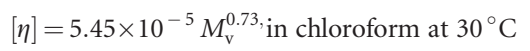

where $[\eta]$ is expressed in $\mathrm{dlg}^{-1}$. The specific rotation for PDLA and PLLA was $+151.7^{\circ}$ and $-150.3^{\circ}$, respectively, indicating that they are composed of $96.7 \%$ D-lactide and $95.7 \%$ L-lactide. $^{11}$

\section{Preparation of the cast film and analysis of the morphology}

PLLA (or PDLA) was dissolved in CHL, ACN or THF at ambient temperature $\left(\sim 20^{\circ} \mathrm{C}\right)$ for the $\mathrm{CHL}$ and THF solution, and at $40^{\circ} \mathrm{C}$ for the ACN solution, so that the polymer concentration $C_{\mathrm{p}}(1 \mathrm{wt} / \mathrm{v} \%)$ will be smaller than the critical polymer concentration $C_{\mathrm{p}}{ }^{*}(\sim 1.4 \mathrm{wt} / \mathrm{v} \%)$, the point at which polymer chains start to overlap each other. Then, the solution of PDLA and the solution of PLLA, which were dissolved in a common solvent, were mixed in equal volume-ratios. The mixture was stirred for $2 \mathrm{~h}$ and cast on a petri dish at ambient temperature $\left(\sim 20^{\circ} \mathrm{C}\right)$. The film thus prepared was annealed for $2 \mathrm{~h}$ at $180^{\circ} \mathrm{C}$, which is above the melting temperature ${ }^{3}$ of crystalline PDLA or PLLA $\left(\sim 170^{\circ} \mathrm{C}\right)$ and well below the melting temperature ${ }^{3}$ of crystalline sc-PLA $\left(210-230^{\circ} \mathrm{C}\right)$. The mix-PLA films prepared with the cast-solvents CHL, ACN and THF are hereafter referred to as mix-PLA(CHL), mix-PLA(ACN) and mix-PLA(THF), respectively. The PLLA film, a reference sample, was prepared with cast-solvent CHL, which is referred to as PLLA(CHL).

The morphology of the cast films was analyzed by DSC (DSC-6100 Seiko Instruments Inc., Chiba, Japan), WAXD (X'Pert PRO MPD,PANalytical, Tokyo, Japan) and SAXS. WAXD and SAXS were carried out by using, as an $\mathrm{X}$-ray source, the $\mathrm{CuK} \alpha$ line (wavelength $\lambda$ is $0.1541 \mathrm{~nm}$ ) and synchrotron orbital radiation ( $\lambda$ is $0.1488 \mathrm{~nm}$ ), respectively, set up in the photon factory of the high energy Accelerator Organization at Tsukuba, Ibaraki, Japan. The scattered intensity $I(q)$ is expressed as the magnitude of the scattering vector, $q$, which is defined by $(4 \pi / \lambda) \sin (\theta)$, where $2 \theta$ is a scattering angle. The details of the instrumentation for the synchrotron radiation are described elsewhere. ${ }^{12,13}$

\section{Analysis of the structure of mix-PLA in solution and conformational analysis of PLLA in solution}

The elapsed time dependence of the mix-PLA structure after being dissolved in the cast-solvent was observed from a change in the transmittance of visible light $(\mathrm{He}-\mathrm{Ne}$ laser light, wavelength $=632.8 \mathrm{~nm})$ passing through the solution and also from a change of its SAXS curve. Moreover, the chain conformation of isolated PLLA dissolved in the cast-solvent was measured from SAXS. In all of these measurements, the polymer concentration $C_{\mathrm{p}}(\sim 1 \mathrm{wt} / \mathrm{v} \%)$ was kept unchanged during the measurements. SAXS measurements were carried out in the photon factory, as was stated above.

\section{RESULTS AND DISCUSSION}

Photographs are shown in Figure 1 of the appearance of (a) mixPLA(CHL), (b) mix-PLA(ACN) and (c) mix-PLA(THF). As is easily observed, mix-PLA(ACN) and mix-PLA(THF) are opaque, and mix-PLA(CHL) is transparent. Moreover, mix-PLA(CHL) and mixPLA(ACN) are tough, but mix-PLA(THF) is brittle and breakable. Such film features seem to reflect a difference in their morphology.

Figure 2 shows DSC curves of mix-PLA films and the reference PLLA(CHL) sample, which were registered by scanning at a heating rate of $5^{\circ} \mathrm{C} \mathrm{min}^{-1}$. In mix-PLA films, an endothermic peak in the range of $210-230^{\circ} \mathrm{C}$ appears due to melting ${ }^{2}$ of the sc-PLA crystal, but no peak appears in the range of $155-175^{\circ} \mathrm{C}$ due to melting ${ }^{2}$ of the PLLA or PDLA crystal. The melting temperature of sc-PLA in mix-PLA(CHL), $225-235^{\circ} \mathrm{C}$, is a little higher than that of sc-PLA in mix-PLA(ACN) and mix-PLA(THF). This shows that the crystal size of sc-PLA in mix-PLA(CHL) may be a little larger.

The absence of crystalline PLLA and crystalline PDLA in mix-PLA films was also confirmed by WAXD patterns in Figure 3 for (a) mixPLA(CHL), (b) mix-PLA(ACN), (c) mix-PLA(THF) and (d) PLLA(CHL). The mix-PLA films show diffraction peaks of sc-PLA at $2 \theta$ diffraction angles of $12^{\circ}, 21^{\circ}$ and $24^{\circ}$, which are assigned ${ }^{14}$ to (110),(300)/(030) and (220), respectively. There are no diffraction peaks due to PLLA crystals.

Marubayashi et al. ${ }^{9}$ have reported that when a PLLA amorphous film is exposed to solvent vapor or immersed in solvent in the liquid state in a temperature range of -25 to $25^{\circ} \mathrm{C}$, PLLA forms a complex crystal ( $\varepsilon$-form) with the solvent. Their experimental conditions are quite different from the present one for preparing a cast film of mixPLA. Equal amounts of PDLA and PLLA were mixed in a dilute solution at ambient temperature, $\sim 20^{\circ} \mathrm{C}$, and cast at that temperature. Then, the film thus prepared was annealed for $2 \mathrm{~h}$ at $180^{\circ} \mathrm{C}$. Therefore, the possibility that the complex of PLLA or PDLA with solvent may be formed in the present cast process is quite low. In fact, there were no detectable diffraction peaks due to these complexes with the solvent in Figure 3.

The DSC curves of mix-PLA(ACN) and mix-PLA(THF) (Figure 2) have another endothermic peak in the range of $190-205^{\circ} \mathrm{C}$. Furuhashi et al. ${ }^{15}$ suggested that the peak is due to imperfect sc-PLA that has a structure that is slightly modified from the intact structure of sc-PLA. Moreover, the WAXD patterns (Figure 3) of mix-PLA(ACN) and mix-PLA(THF) show another diffraction peak in the range of $30-35^{\circ}$, and the shape of the diffraction peak at $\sim 21^{\circ}$ is slightly deformed compared with the corresponding one in mix-PLA(CHL). These data indicate that imperfect sc-PLA exists in mix-PLA(ACN) and mixPLA(THF).

The imperfect sc-PLA would be more or less characterized by transient morphology, such as a deformed crystal structure and a short lamellar thickness, and so it is probable that the morphology of mix-PLA(ACN) and mix-PLA(THF) might be further modified in a different way by annealing the films at 140,180 or $220^{\circ} \mathrm{C}$ for several hours. Figure 4 shows the relation between the SAXS scattered intensity $I(q)$ and $q$ for the annealed films, (a) mix-PLA(CHL), (b) mix-PLA(ACN) and (c) mix-PLA(THF). All scattering curves have a hump in the $q$ range from $0.2-0.8 \mathrm{~nm}^{-1}$, and the abscissa position of the hump, $q^{*}$, shifts to a smaller value as $T$ is raised from $140-220^{\circ} \mathrm{C}$. That is, it shifts from $0.45 \mathrm{~nm}^{-1}$ at $140{ }^{\circ} \mathrm{C}$ to $0.35 \mathrm{~nm}^{-1}$ at $180^{\circ} \mathrm{C}$ and then to $0.325 \mathrm{~nm}^{-1}$ at $220^{\circ} \mathrm{C}$ for mix-PLA(CHL), from $0.7-0.60 \mathrm{~nm}^{-1}$ and then to $0.425 \mathrm{~nm}^{-1}$ for mix-PLA(ACN), and from $0.65-0.60 \mathrm{~nm}^{-1}$ and then to $0.25 \mathrm{~nm}^{-1}$ for mix-PLA(THF).

Taking into account many studies ${ }^{2,4-7}$ on the morphological behavior of sc-PLA, it seems proper to assume that the hump in 

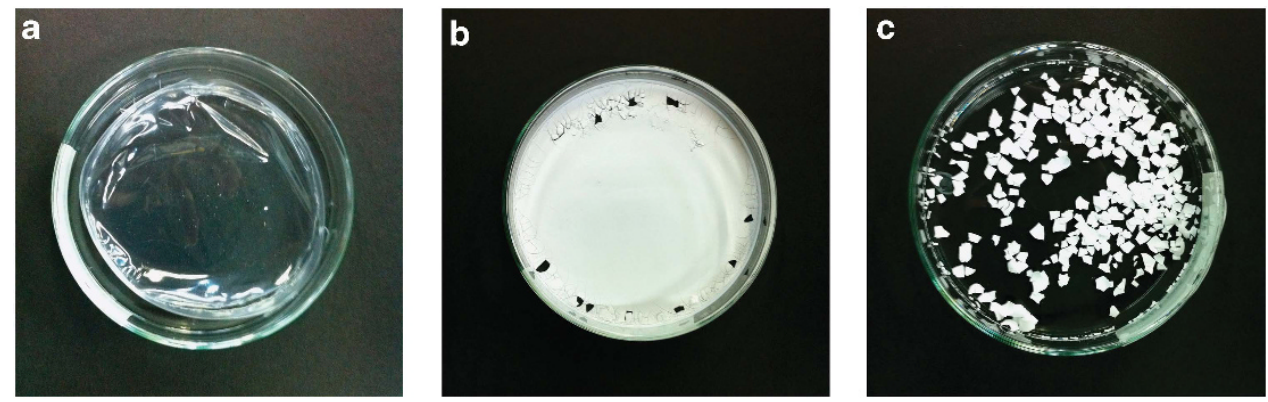

Figure 1 Appearance of cast films prepared with different cast-solvents: (a) mix-PLA(CHL), (b) mix-PLA(ACN) and (c) mix-PLA(THF),

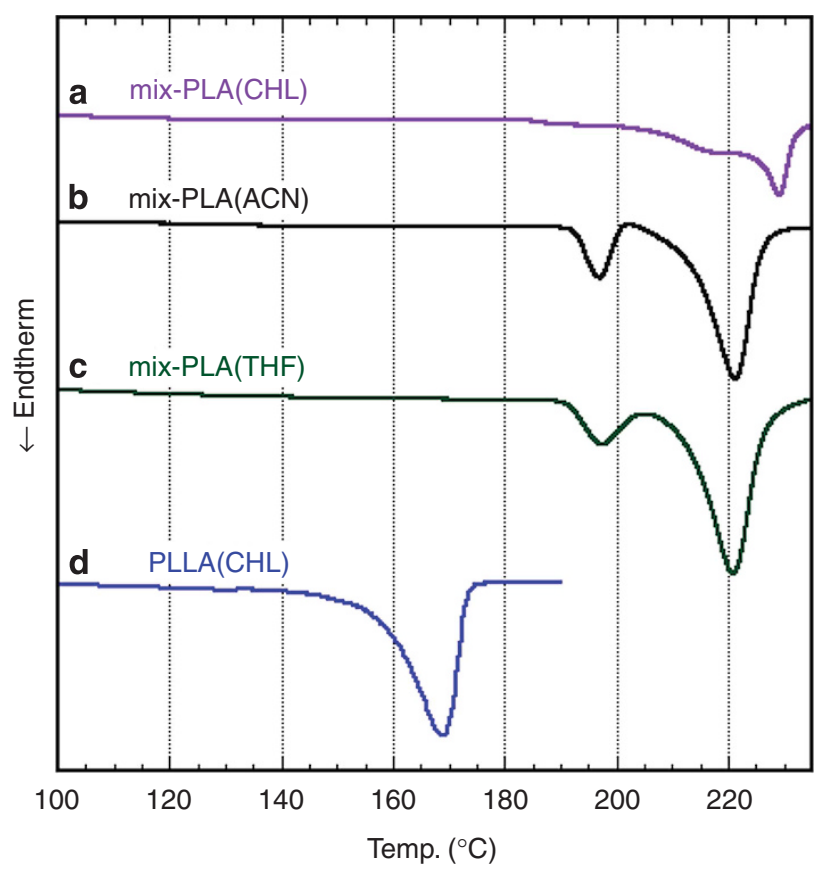

Figure 2 DSC curves of sc-PLA cast films prepared with different castsolvents: (a) mix-PLA(CHL), (b) mix-PLA(ACN), (c) mix-PLA(THF) and (d) PLLA(CHL).

the SAXS curves of the cast films might be due to the lamella structure of sc-PLA. Therefore, assuming that $q^{*}$ is related to the lamellar thickness, $d$, by Equation (2),

$$
d=2 \pi / q *
$$

it can be derived that $d$ shifts in the following manner- $14.0 \mathrm{~nm} \rightarrow$ $18.0 \mathrm{~nm} \rightarrow 19.3 \mathrm{~nm}, \quad 9.0 \mathrm{~nm} \rightarrow 10.5 \mathrm{~nm} \rightarrow 14.8 \mathrm{~nm}$ and $9.7 \mathrm{~nm} \rightarrow$ $10.5 \mathrm{~nm} \rightarrow 25.1 \mathrm{~nm}$, respectively-as $T$ is raised from $140-220^{\circ} \mathrm{C}$. These data indicate that the annealing treatment would promote lamellar thickening for all samples, and the morphology of mix-PLA(ACN) and mix-PLA(THF) is more labile compared with mix-PLA(CHL).

Such a difference in the liability of their morphology might also manifest itself in the morphology produced when the mix-PLA sample is momentarily exposed at $240{ }^{\circ} \mathrm{C}$, a temperature slightly higher than the melting temperature of sc-PLA crystals $\left(210-230^{\circ} \mathrm{C}\right)$. Thus, the sample was heated up from room temperature to $240^{\circ} \mathrm{C}$ at a rate of $5^{\circ} \mathrm{C} \mathrm{min}^{-1}$, and as soon as the temperature of $240^{\circ} \mathrm{C}$ was reached, it was cooled again to room temperature at a rate of $5{ }^{\circ} \mathrm{Cmin}^{-1}$. The DSC curves in Figure 5 were registered during this

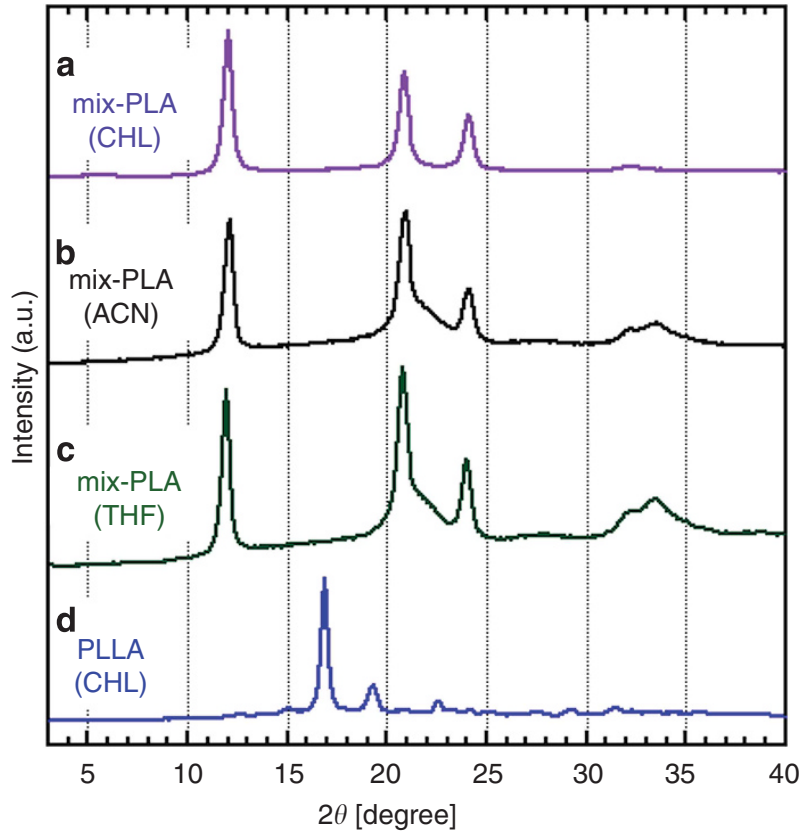

Figure 3 WAXD patterns of Sc-PLA cast films prepared with different cast-solvents: (a) mix-PLA(CHL), (b) mix-PLA(ACN), (c) mix-PLA(THF) and (d) PLLA(CHL).

cooling process. None of the curves coincide with each other, indicating that their morphological states are far from the isotropic melt states.

In the curves of mix-PLA(ACN) and mix-PLA(THF) (Figure 5), exothermic peaks appear in the range of $180-210^{\circ} \mathrm{C}$ and in the range of $120-150^{\circ} \mathrm{C}$, which are due to crystallization of sc-PLA and crystallization of PLLA and PDLA, respectively, but in the DSC curves of mix-PLA(CHL), only one exothermic peak appears due to crystallization of sc-PLA. That is, the imperfect sc-PLA in mixPLA(ACN) and mix-PLA(THF) is reconstructed into crystals of sc-PLA, PDLA and PLLA by this annealing and cooling treatment. Thus, these data reconfirm that the original morphology of mixPLA(ACN) and mix-PLA(THF) is more labile compared with that of mix-PLA(CHL).

Now, the analysis is directed to the problem of determining how the morphology of mix-PLA(ACN), mix-PLA(THF) and mixPLA(CHL) depends on the type of cast-solvents employed. Here, it is assumed that the structure of sc-PLA would be constructed by the balance of interaction energy $\mathrm{E}_{1}$ between PLLA and PDLA chains in solution, interaction energy $\mathrm{E}_{2}$ between PLLA chains and solvent 
a

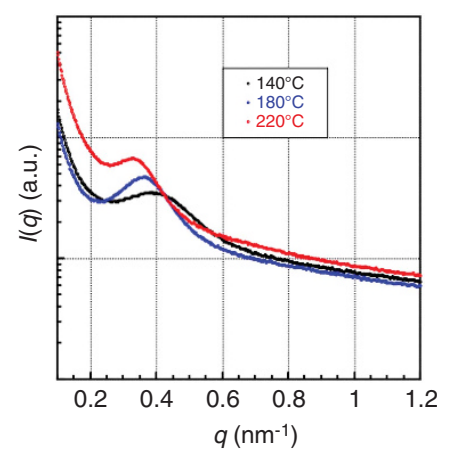

b

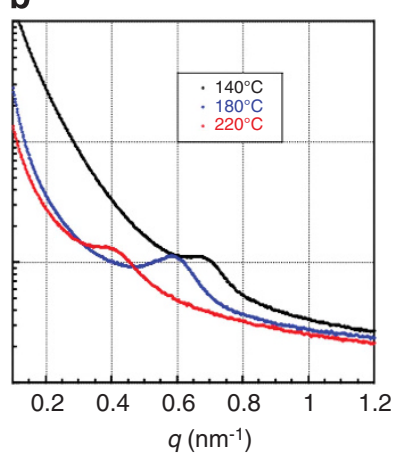

C

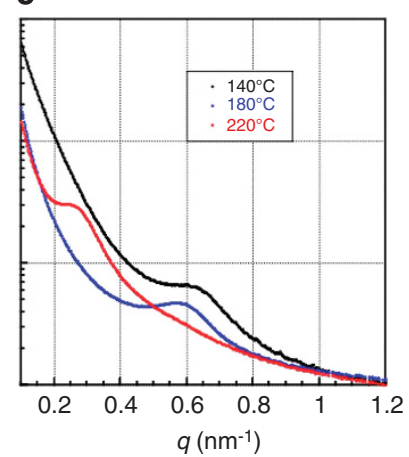

Figure 4 Thermal treatment temperature dependence of SAXS curves of (a) mix-PLA(CHL), (b) mix-PLA(ACN) and (c) mix-PLA(THF).

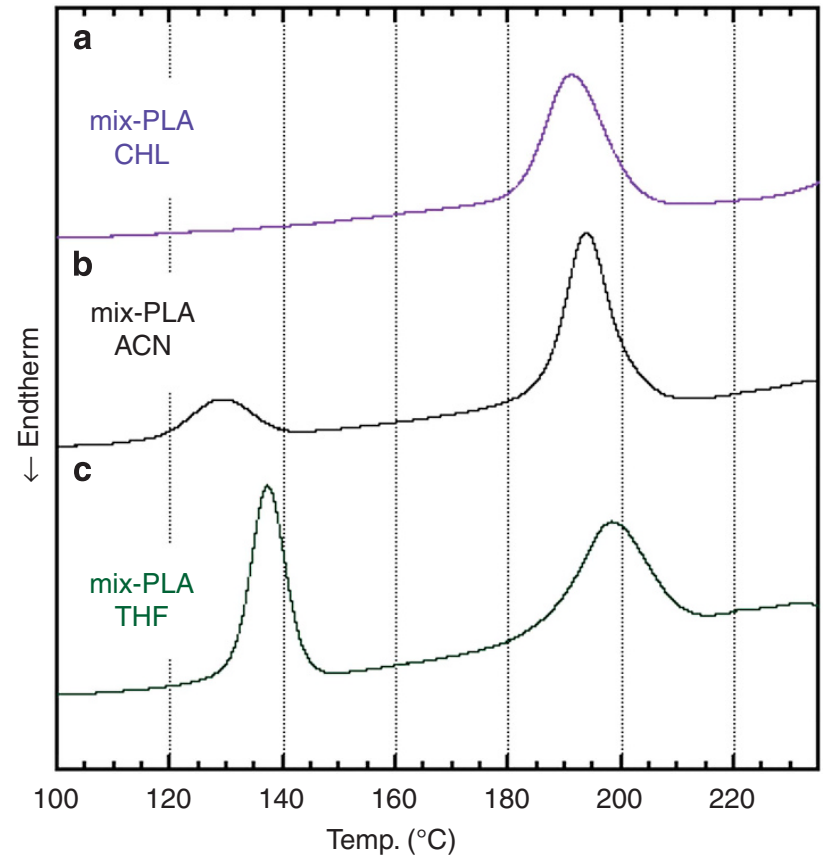

Figure 5 DSC curves in the cooling process of Sc-PLA cast films prepared with different cast-solvents: (a) mix-PLA(CHL), (b) mix-PLA(ACN), (c) mixPLA(THF) and (d) PLLA(CHL).

molecules, interaction energy $\mathrm{E}_{3}$ between PDLA chains and solvent molecules and interaction energy $\mathrm{E}_{4}$ between PLLA and PDLA chains in the crystal lattice. It is reasonable to assume that $E_{2}=E_{3}$ and that $\mathrm{E}_{4}$ is independent of the type of solvent. Consequently, it follows that the balance between $E_{1}$ and $E_{2}$ brings about the solvent effect on the morphology.

With the relation between $E_{1}$ and $E_{2}$, the scheme for the formation of sc-PLA is classified into two categories, Category-I and -II. In Category-I, the relation $\mathrm{E}_{1}>\mathrm{E}_{2}$ holds over the entire concentration range. The aggregation of PLLA and PDLA chains will start in the dilute solution, and a change of the original conformations to helical ones will simultaneously or successively follow after their aggregation. Moreover, the change rate will significantly depend on the chain conformation in dilute solution. In Category-II, in contrast, the relation $\mathrm{E}_{1}<\mathrm{E}_{2}$ holds over a range from dilute to moderate

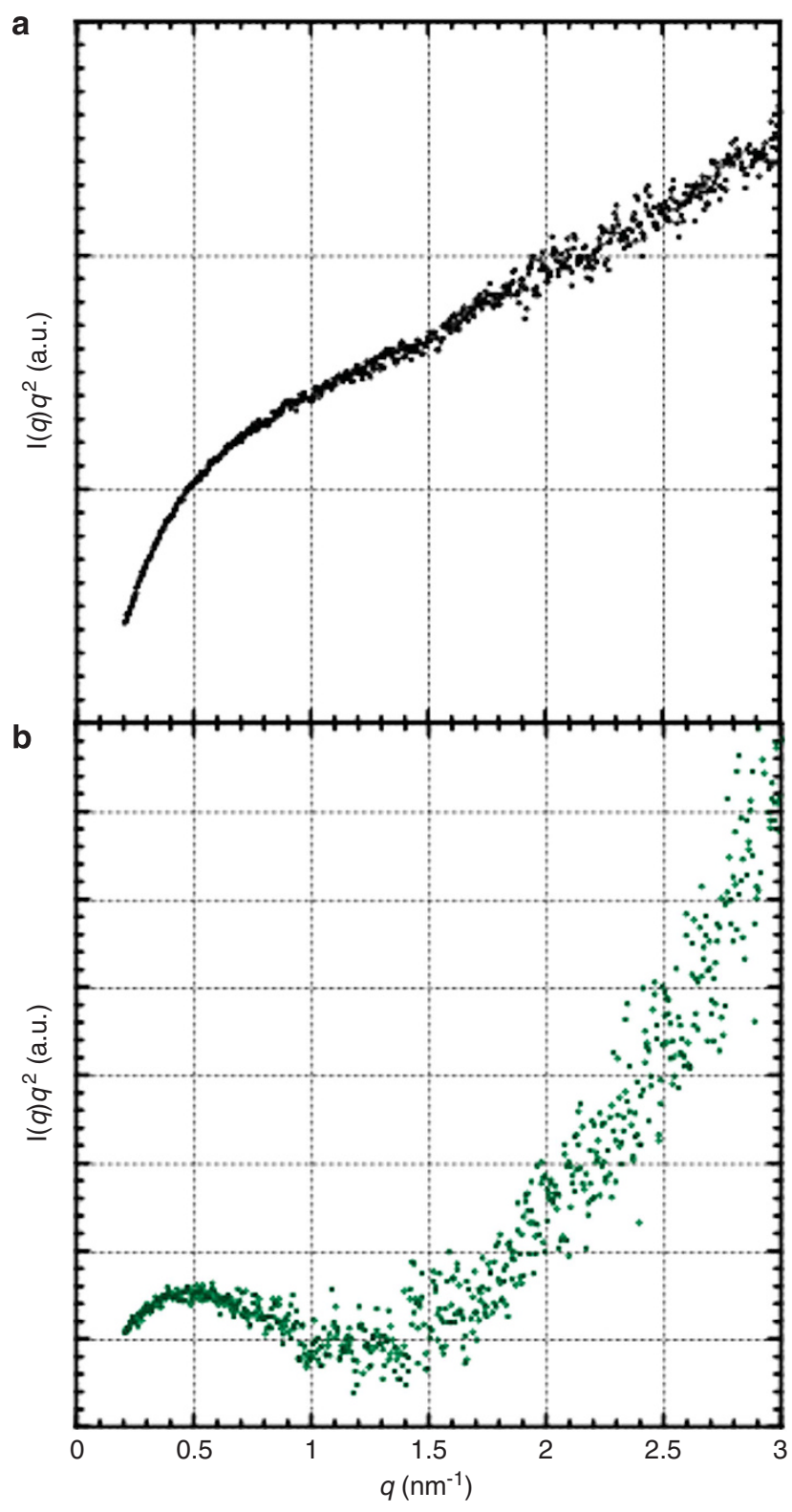

Figure 6 Kratky plot for PLLA dissolved in (a) ACN and (b) THF. 
concentrations. In this case, their aggregation and changes in the original chain conformations will be retarded until a state involving a higher polymer concentration is attained.

For the sake of obtaining some hints to solve the problem, the original chain conformation of PLLA and PDLA in cast-solvents and the aggregation rate of a mixture of PLLA and PDLA in solution were analyzed below. In Figure 6, SAXS data for PLLA $\left(C_{\mathrm{p}}: 1 \mathrm{wt} / \mathrm{v} \%\right)$ dissolved in (a) ACN and (b) THF is represented in the form of $I(q) q^{2}$ vs $q$, the so-called Kratky plot. SAXS data for the CHL solution were not available because the signal-to-noise ratio is too small for $I(q)$ to be analyzed. It is clearly shown that $I(q) q^{2}$ in (a) monotonically increases as $q$ is increased, whereas $I(q) q^{2}$ in (b) has a hump in the $q$ range from $0.2-1.0 \mathrm{~nm}^{-1}$. As was shown in our study, ${ }^{16}$ these characteristic curves in (a) and (b) can be mimicked by the scattering function of wormlike chains and globular coils, respectively. Thus, it is supposed that PLLA and PDLA would take the form of an expanded chain in ACN solution and a compact globular coil in THF solution. Needless to say, it will be easier for an expanded chain to recoil into the helical chain than for a globular coil.

The aggregation rate of a mixture of PLLA and PDLA in solution was measured from the elapsed time dependence of the transmittance

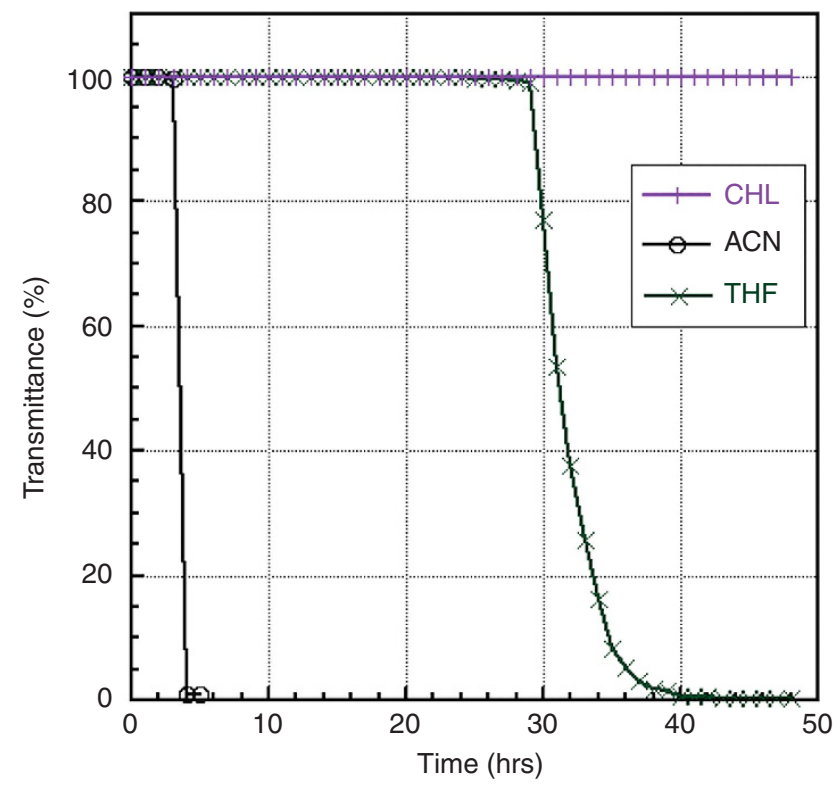

Figure 7 Time dependence of the transmittance of visible light through solutions of mix-PLA dissolved in ACN and in THF. of visible light passing through the mix-PLA solution, in which measurement of the elapsed time started immediately after the PLLA and PDLA were mixed. As is shown in Figure 7, the ACN solution of mix-PLA $\left(C_{\mathrm{p}}: 1 \mathrm{wt} / \mathrm{v} \%\right)$ and the THF solution become turbid in $\sim 4 \mathrm{~h}$ and $30 \mathrm{~h}$, respectively, whereas the $\mathrm{CHL}$ solution remained transparent for at least 3 days. When PLLA is dissolved in ACN or THF, the solution remains transparent for the same amount of time. These data indicate that the decrease in the transmittance of light is due to formation of aggregates consisting of PLLA and PDLA chains, which leads to the formation of sc-PLA in the concentrated solution. Aggregates are more rapidly constructed in the ACN solution than in the THF solution or in the CHL solution.

The aggregation rate of a mixture of PLLA and PDLA in solution $\left(C_{\mathrm{p}}: 1 \mathrm{wt} / \mathrm{v} \%\right)$ was also estimated from a change in their SAXS curves. The SAXS curve for the ACN solution of mix-PLA (Figure 8a) began to exhibit a hump in the $q$ range below $\sim 0.4 \mathrm{~nm}^{-1}$ after $2 \mathrm{~h}$, while the SAXS curve in Figure $8 \mathrm{~b}$ for the THF solution of mix-PLA remained unchanged for $4 \mathrm{~h}$. In contrast, the ACN solution of PLLA (Figure 8c) had no hump in the curve, which is similar to the results shown in Figure 8b. As humps in SAXS curves for the suspension would reflect the interference between aggregates, it is clarified again that the aggregates are more rapidly formed in the ACN solution than in the THF solution. However, it remains unresolved whether the aggregate of PLLA and PDLA involves sc-PLA. For this purpose, the circular dichroism spectrum was measured for a suspension containing equal amounts of PLLA and PDLA, but it produced a basically horizontal line over the relevant range.

In Figure 6, it was stated that PLLA and PDLA take on the form of an expanded chain in the ACN solution and a compact globular coil in THF solution, and so it was suggested that the conformational change of PLLA and PDLA in dilute solutions to the helical forms would occur faster in the ACN solution than in the THF solution. In Figures 7 and 8, moreover, it is shown that the aggregation of PLLA and PDLA will start even in a dilute solution and that the aggregation rate is faster in the ACN solution than in the THF solution. From these data, it is suggested that Category-I would be proper as the scheme when sc-PLA is formed by casting a THF or ACN solution of mix-PLA. In this case, because of the rapid formation of sc-PLA, the structure would tend to involve imperfect sc-PLA, as is shown in Figures 2 and 3.

As is shown in Equation (1), the PLLA and PDLA in the CHL solution behave as expanded chains. Nevertheless, the aggregation rate of PLLA and PDLA is quite slow in the CHL solution, as is shown in Figure 7. The observations seem to indicate that the solubility of expanded and/or helical PLLA and PDLA in the CHL solution is

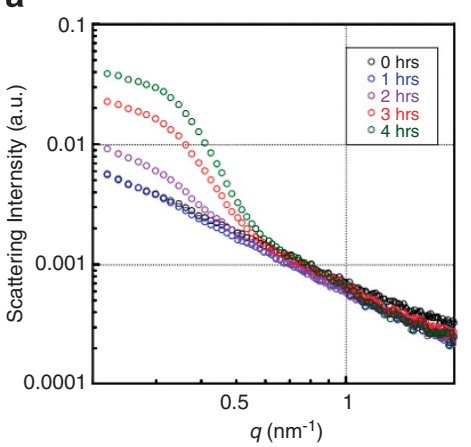

b

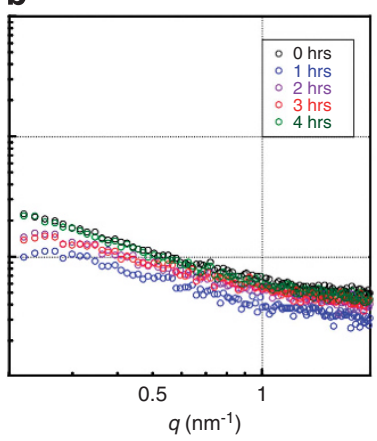

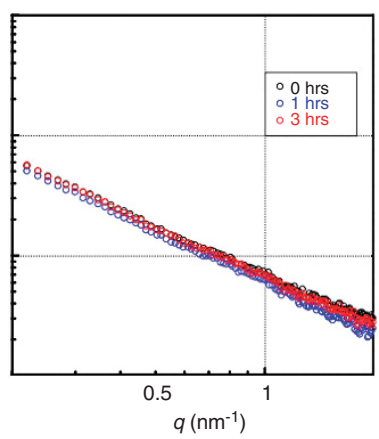

Figure 8 Time dependence of SAXS profiles for (a) mix-PLA in ACN, (b) mix-PLA in THF and (c) PLLA in ACN. 
much better than in the ACN solution or in the THF solution, and so the rate of their aggregation is extremely retarded. Thus, it is suggested that Category-II would be proper as the scheme when sc-PLA is formed by casting the CHL solution of mix-PLA. At such a slow aggregation rate, sc-PLA would be structured without the interference of excess PLLA and PDLA and/or occasional pairing or aggregation of their short helical sequences. This may be the reason why the crystal structure of sc-PLA in mix-PLA(CHL) is basically intact, as is shown in Figures 2 and 3.

\section{CONCLUSIONS}

Semicrystalline films were prepared by casting CHL, ACN or THF solutions of a mixture of PLLA and PDLA. From the analysis by DSC, WAXD and SAXS, it was clarified that all of the films involve a sc-PLA of PLLA and PDLA, but no crystals of PLLA or PDLA, and the morphology significantly depends on the cast-solvent. The origin for the marked cast-solvent dependence of the morphology was studied by assuming that the structure of sc-PLA would be constructed by the balance of interaction energy $E_{1}$ between PLLA and PDLA chains in solution and interaction energy $\mathrm{E}_{2}$ between PLLA or PDLA chains and solvent molecules. The scheme for the formation of sc-PLA was classified into two categories, Category-I and -II. In Category-I, the relation $E_{1}>E_{2}$ holds over an entire concentration range, and in Category-II, the relation $\mathrm{E}_{1}<\mathrm{E}_{2}$ holds over a range from dilute to moderate concentrations. The data indicate that Category-I would be proper when sc-PLA is formed by casting the THF or ACN solution of mix-PLA and that Category-II would be appropriate when sc-PLA is formed by casting the CHL solution of mix-PLA.

This work was performed under the approval of the Photon Factory Program Advisory Committee (proposal nos. 2011G070 and 2011G509).

\section{CONFLICT OF INTEREST}

The authors declare no conflict of interest.

\section{ACKNOWLEDGEMENTS}

Our deepest appreciation is given to Dr Kurita for valuable discussions and to Dr Igarashi and Dr Shimizu of the High Energy Accelerator Research

Organization and Professor Suzuki and Dr Kurumi of Electrical Engineering,
Nihon University, for technical support. We would also like to thank Creative Materials Research Center (Research Institute of Science \& Technology, Nihon University) for the use of the analytical apparatus. This work was supported in part by Nihon University Strategic Projects for Academic Research, 'Nanomaterial-based Photonic, Quantum and Bio Technologies.'

1 Tsuji, H. Poly(lactide) stereocomplexes: formation, structure, properties, degradation, and applications. Macromol. Biosci. 5, 569-597 (2005).

2 Tsuji, H. \& Ikada, Y. Stereocomplex formation between enantiomeric poly(lactic acid)s. XI. Mechanical properties and morphology of solution-cast films. Polymer 40, 6699-6708 (1999).

3 Ikada, Y., Jamshidi, K., Tsuji, H. \& Hyon, S. -H. Stereocomplex formation between enantiomeric poly( lactides). Macromolecules 20, 906-908 (1987).

4 Brizzolara, D., Cantow, H. -J., Diederichs, K., Keller, E. \& Domb, A. J. Mechanism of the stereocomplex formation between enantiomeric poly(lactide)s. Macromolecules 29 191-197 (1996).

5 Zhang, J., Tashiro, K., Tsuji, H. \& Domb, A. J. Investigation of phase transitional behavior of poly(L-lactide)/ poly(D-lactide) blend used to prepare the highly-oriented stereocomplex. Macromolecules 40, 1049-1054 (2007).

$6 \mathrm{He}$, Y., Xu, Y., Wei, J., Fan, Z. \& Li, S. Unique crystallization behavior of poly(L-lactide)/ poly(D-lactide) stereocomplex depending on initial melt states. Polymer 49, 5670-5675 (2008).

7 Fujita, M., Sawayanagi, T., Abe, H., Tanaka, T., Iwata, T., Ito, K., Fujisawa, T. \& Maeda, M. Stereocomplex formation through reorganization of poly(L-lactic acid) and poly(Dlactic acid) crystals. Macromolecules 41, 2852-2858 (2008).

8 Ajioka, M., Enomoto, K., Suzuki, K. \& Yamaguchi, A. Basic properties of polylactic acid produced by the direct condensation polymerization of lactic acid. Bull. Chem. Soc Jpn. 68, 2125-2131 (1995).

9 Marubayashi, H., Asai, S. \& Sumita., M. Complex crystal formation of poly(L-lactide) with solvent molecules. Macromolecules 45, 1384-1397 (2012).

10 Schindler, A. \& Harper, D. Polylactide 11 viscosity-molecular weight relationships and unperturbed chain dimensions. J. Polym. Sc.Poly. Chem. Ed. 17, 2593-2599 (1979).

11 Tonelli, E. A. \& Flory, P. J. The configuration statistics of random poly (lactic acid) chains. I. experimental results. Macromolecules 3, 225-227 (1969).

12 Ueki, T., Hiragi, Y., Izumi, Y., Tagawa, H., Kataoka, M., Muroga, Y., Matsushita, T. \& Amemiya, Y. Photon Factory Activity Report V170-V171 (1982-1983).

13 Muroga, Y., Muraki, T., Noda, I., Tagawa, H., Holtzer, A. \& Holtzer, M. E. Chain unfolding equilibria of. $\alpha$-tropomyosin coiled coils studied by small angle $X$-ray scattering. J. Amer. Chem. Soc. 117, 5622-5626 (1995).

14 Sawai, D., Tsugane, Y., Tamada, M., Kanamoto, T., Sungil, M. \& Hyonwaxs, S.H. Crystal density and heat of fusion for a stereo-complex of poly(L-lactic acid) and poly(D-lactic acid). J. Polym. Sci. Polym. Phys. Ed. 45, 2632-2639 (2007).

15 Furuhashi, Y., Kimura, Y., Yoshie, N. \& Yamane, H. Higher-order structures and mechanical properties of stereocomplex-type poly(lactic acid) melt spun fibers. Polymer 47, 5965-5972 (2006).

16 Muroga, Y., Iida, S., Shimizu, S., Ikake, H. \& Kurita, K. Conformation of poly(sodium ethacrylate) in solution studied by small-angle X-ray scattering. Biophys. Chem. 110, 49-58 (2004). 